\title{
La supervisión educativa. Funciones gerenciales para garantizar la eficiencia
}

\author{
Educational supervision. Management functions to use efficiency
}

\author{
a) \\ Leida Ayary Flores \\ ayari970@hotmail.com \\ Gobierno del Distrito Capital- \\ Secretaria para la Educación, Venezuela
}

Artículo recibido en septiembre 2018 Arbitrado en octubre 2018

\section{Resumen}

El objetivo de este estudio fue analizar las funciones gerenciales de los supervisores adscritos a los circuitos educativos de la Secretaría para la Educación del Gobierno del Distrito Capital (Caracas, Venezuela), a fin de abordar todo el conjunto de actividades que un supervisor debe realizar durante su gestión educativa. El estudio fue descriptivo, de campo y un proyecto factible. El diseño no experimental. La técnica utilizada fue la encuesta, con un cuestionario dirigido a los directores y docentes de las escuelas seleccionadas. La muestra estuvo conformada por seis distritos escolares, de los cuales se seleccionaron siete unidades educativas con diecisiete directores y ciento veinte docentes de aula. Los resultados evidenciaron que existe un alto porcentaje de debilidades en los procesos gerenciales realizados por los supervisores. Hay ausencia de la aplicación de la acción supervisora y una escasa orientación de los procesos de asesoramiento, planificación, ejecución, organización, motivación, comunicación.

\begin{abstract}
The objective of this study was to analyze the managerial functions of the supervisors assigned to the educational circuits of the Secretariat for Education of the Government of the Capital District (Caracas, Venezuela), in order to address the entire set of activities that a supervisor must carry out during its educational management. The study was descriptive, field and a feasible project. The non-experimental design. The technique used was the survey, with a questionnaire addressed to the principals and teachers of the selected schools. The sample consisted of six school districts, from which seven educational units with seventeen principals and one hundred twenty classroom teachers were selected. The results showed that there is a high percentage of weaknesses in the managerial processes carried out by the supervisors. There is absence of the application of supervisory action and little orientation of the processes of advice, planning, execution, organization, motivation, communication.
\end{abstract}

Palabras clave:

gerencia; procesos gerenciales; supervisión; funciones del supervisor; acción supervisora

\section{Keywords:}

management, management processes, supervision, supervisor functions, supervisory action 
INTRODUCCIÓN

A nivel mundial todos los escenarios están experimentando cambios producto de la influencia de los grupos sociales y las organizaciones que trabajan a favor del desarrollo de la población (Borjas y Vera, 2008). De allí que las instituciones educativas tengan que hacer ajustes, con el fin de satisfacer las necesidades que son producto de esas transformaciones, ya que gracias a ella se logra el desarrollo científico, tecnológico y económico de la sociedad.

Por ello, en Venezuela, la educación amerita cambios que permitan atender la demanda científica y tecnológica de los últimos tiempos, y para lograrlos se requiere de profesionales que estén acordes con las nuevas políticas y esquemas que necesita el país (Maldonado, 2008), debido a que estos cambios tienen como fin lograr una mejor calidad educativa, la cual debe partir de la discusión y el consenso entre los principales actores del proceso educativo. Estos cambios también deben reflejarse en la supervisión educativa, la cual busca, en principio, controlar y asegurar la ejecución y el cumplimiento de los procesos de enseñanza y aprendizaje, así como la capacitación y el crecimiento profesional de los docentes quienes tienen la misión de educar para lograr una sociedad más justa y humana enmarcada dentro de un mundo de grandes cambios.

La supervisión es un proceso dinámico y sistemático que permite garantizar el cumplimiento de los fines y propósitos del Estado en sus políticas educativas. Su misión es conducir, orientar, asesorar, controlar, coordinar y evaluar mediante una acción participativa y cooperativa, los cambios y transformaciones que se dan para mejorar la educación. De allí, que la acción supervisora requiera de atención y además de una orientación dirigida al docente para que este logre resolver los problemas concretos, que no esté centrada en una práctica de vigilancia que solo busca aplicar correctivos superficiales, sino que evalué y mejore los factores que afectan a la organización y a los procesos de enseñanza y aprendizaje.

En este sentido, Ruiz (2005) afirma que los problemas que ocasiona el sistema educativo se deben, entre otras causas, a que la supervisión no toma en cuenta las verdaderas necesidades y problemas que viven las instituciones educativas, por la falta de planificación, asesoramiento, seguimiento y control de los procesos.

Para Bringuier (2001) "la Supervisión Educativa debe ser concebida como un proceso de investigación permanente, debe realizarse profundizando en los problemas, en sus causas, considerando no solo resultados, sino los procesos y el valor representativo de las muestras" (p. 16).

Por tanto, la problemática de la supervisión educativa en Venezuela, se manifiesta en el hecho de que no cumple su función de manera eficiente y no asesora a los docentes directivos, docente de aula en el antes, durante y después del ejercicio, dándoles las herramientas necesarias que les sirvan de apoyo para la solución de sus problemas (Mogollón, 2008; Ruiz, 1992; Ruiz, 2005). Esto implica que el personal supervisor no ha mostrado capacidad y formación para dirigir y controlar los procesos educativos y no se ajusta al sistema escolar, al actuar de manera incoherente en su gestión gerencial, por desconocimiento, por el rechazo a los nuevos paradigmas o por falta de iniciativa en la aplicación de sus funciones gerenciales, por cuanto es necesario prestar atención técnica y profesional para promover el rendimiento de la organización educacional.

Para Chiavenato (2006), la función gerencial busca alcanzar la calidad, a partir del uso racional que debe hacerse de los recursos involucrados, lo que a su vez va a 
determinar el logro de los objetivos planteados por una organización, que en estecaso es una institución educativa. Para este autor "la gerencia se refiere a las organizaciones que efectúan actividades de planificación, organización, dirección y control, a objeto de utilizar sus recursos humanos, físicos y financieros con la finalidad de alcanzar objetivos, comúnmente relacionados con beneficios económicos" (p. 146).

La supervisión, en el ámbito educativo, está circunscrita a los cargos directivos de las instituciones educativas y a ciertos cargos de las entidades administrativas, lo que lleva a afirmar que está constituye una función dentro de la estructura organizativa del sector educativo. Esto ha llevado a considerar que las principales acciones que esta debe emprender son: evaluar, asesorar y controlar (Lemus, 2005). Sin embargo, hay quienes consideran que esta puede ser aplicada como un método, como una disciplina, como un sistema y como contraloría social (Mendoza, 2018).

Según Ayala (2014), existen normas y principios que deben ser considerados durante la supervisión educativa, con el fin de que se garantice la eficiencia y eficacia. Son estos: a) el acompañamiento, el cual busca más que fiscalizar garantizar el cumplimiento de los estándares de calidad; b) la horizontalidad, que busca un clima de respeto y responsabilidad entre el supervisor y el supervisado, basado en las buenas relaciones entre todos los miembros de la institución educativa; c) la objetividad, imparcialidad y transparencia cuando al momento de emitir juicios; d) la profesionalización, especialización y dignificación de la función del docente.

Por otra parte, Esteban (2019) presenta tres perspectivas que permiten explicar el término supervisión educativa. La primera es la bibliográfico-histórica, referida al enfoque centrado en la ayuda y estímulo por parte de directores e inspectores, el cual prevaleció durante los años 60 y 70 del siglo pasado. La segunda perspectiva está centrada en el plano legal, a partir de la introducción del término en el año 1965. Allí se asignaron tareas de supervisión, dirección y orientación a los inspectores. La tercera perspectiva es la representación conceptual en la que se la ha relacionado con términos tales como: inspección, asesoramiento y evaluación. La cuarta perspectiva es el enfoque desde otras áreas del conocimiento, ya que se ha relacionado con la administración en organizaciones y empresas referidas a la gestión de servicios y a la producción.

Por lo antes expuesto, el objetivo de la presente investigación fue analizar las funciones gerenciales de los supervisores educativos, adscritos a los circuitos educativos de la Secretaría para la Educación del Gobierno del Distrito Capital (Caracas, Venezuela), enmarcadas en las dimensiones: personal, profesional e institucional, cada una con sus respectivos indicadores, a fin de abordar todo el conjunto de actividades que un supervisor debe realizar durante su gestión educativa para poder garantizar el éxito de su rol.

\section{MÉTODO}

La investigación se enmarcó en la modalidad de proyecto factible que según la Universidad Pedagógica Experimental Libertador (UPEL, 2017) es:

la investigación, elaboración y desarrollo de una propuesta de un modelo operativo viable para solucionar problemas, requerimientos o necesidades de organizaciones o grupos sociales; puede referirse a la formulación de políticas, programas, métodos o procesos. El Proyecto debe tener apoyo en una tipo documental, de campo o un diseño que incluya ambas modalidades. (p. 21) 
Esta modalidad ofreció soluciones viables al problema planteado, en este caso las debilidades observadas en la acción supervisora en las diferentes instituciones educativas de las escuelas adscritas al Gobierno del Distrito Capital.

Se inscribió en una investigación de campo de tipo descriptiva, que de acuerdo con Arias (2006) "consiste en la caracterización de un hecho, fenómeno, individuo o grupo con el fin de establecer su estructura o comportamiento" (p. 24). El diseño no experimental, porque el investigador no influyó sobre las variables para manipularlas. Es transversal o transeccional, ya que la información en la recolección de datos se obtuvo en un tiempo único y en un momento dado.

Se consideraron dos variables (ver Tabla 1):

Tabla 1. Operacionalización de variables

\begin{tabular}{|c|c|c|c|}
\hline Variable & $\begin{array}{l}\text { Definición } \\
\text { conceptual }\end{array}$ & Dimensiones & Indicadores \\
\hline Necesidad & $\begin{array}{l}\text { Manifestación natural de } \\
\text { sensibilidad interna que despierta } \\
\text { una tendencia a cumplir un acto o } \\
\text { buscar una determinada categoría } \\
\text { de objetos. }\end{array}$ & Personal & $\begin{array}{l}\text {-Liderazgo } \\
\text {-Motivación } \\
\text {-Comunicación } \\
\text {-Manejo de conflicto } \\
\text {-Relaciones } \\
\text { interpersonales }\end{array}$ \\
\hline supervisores & & Profesional & $\begin{array}{l}\text {-Planificación } \\
\text {-Organización } \\
\text {-Control } \\
\text {-Ejecución } \\
\text {-Toma de decisiones }\end{array}$ \\
\hline $\begin{array}{l}\text { Importancia de } \\
\text { las funciones } \\
\text { gerenciales }\end{array}$ & $\begin{array}{l}\text { Es la cualidad de lo importante, de } \\
\text { lo que es muy conveniente o } \\
\text { interesante, o de mucha entidad o } \\
\text { consecuencia con respecto a las } \\
\text { funciones gerenciales, en este caso } \\
\text { de un supervisor educativo. }\end{array}$ & Institucional & $\begin{array}{l}\text {-Sistema } \\
\text { de recompensa } \\
\text {-Evaluación } \\
\text { de desempeño }\end{array}$ \\
\hline
\end{tabular}

La población se conformó por los docentes y directivos de las 103 escuelas adscritas de los diferentes circuitos educativos que dependen de la Secretaria para la Educación del Gobierno del Distrito Capital, con un total 3890 docentes y 192 directivos entre directores y subdirector. De la población se extrajo una muestra tanto de las escuelas, como de docentes. Las escuelas se estratificaron por parroquia en total seis, de estas se tomaron una o dos por parroquia atendiendo el nivel y la modalidad. Así se pudo obtener una información heterogénea, de las escuelas se seleccionó la muestra de docentes a través del muestreo accidental o casual, el cual según Hurtado y Toro (1998), no responde a una planificación previa en cuanto a los sujetos a elegir. El criterio de selección de los sujetos dependió de la posibilidad de acceder a ellos. La muestra fue de 120 docentes y 17 directivos, atendiendo a la accesibilidad de respuestas (ver Tabla 2). 
Tabla 2. Muestra de la investigación

\begin{tabular}{|c|c|c|c|c|c|c|}
\hline $\begin{array}{l}\text { CIRCUITO } \\
\text { ESCOLAR }\end{array}$ & PARROQUIA & PLANTEL & $\begin{array}{c}\mathrm{N}^{\circ} \mathrm{DE} \\
\text { DIRECTIVOS }\end{array}$ & MUESTRA & $\begin{array}{c}\mathbf{N}^{\circ} \text { DE } \\
\text { DOCENTES }\end{array}$ & MUESTRA \\
\hline 1 & El Valle & $\begin{array}{c}\text { ETDR JUAN } \\
\text { ESPAÑA } \\
\text { UED ENRIQUE }\end{array}$ & 3 & 3 & 69 & 25 \\
\hline 1 & La Pastora & $\begin{array}{c}\text { CHAUMER } \\
\text { UED. JOSE LUIS }\end{array}$ & 3 & 3 & 73 & 21 \\
\hline 1 & $\begin{array}{l}\text { La Pastora } \\
\text { El Paraíso, }\end{array}$ & $\begin{array}{c}\text { RAMOS } \\
\text { PREESCOLAR }\end{array}$ & 3 & 3 & 46 & 17 \\
\hline 3 & $\begin{array}{l}\text { La Vega, } \\
\text { San Juan }\end{array}$ & INES PONTE & 2 & 2 & 14 & 10 \\
\hline 4 & $\begin{array}{c}\text { Antímano, } \\
\text { Caricuao, } \\
\text { Macarao } \\
\text { Sucre, }\end{array}$ & $\begin{array}{l}\text { CDET. DEFENSA } \\
\text { CIVIL JORGE } \\
\text { MURAD SAYEEG } \\
\text { UEDB. JOSÉ }\end{array}$ & 1 & 1 & 69 & 19 \\
\hline 1 & 23 de Enero & $\begin{array}{l}\text { GREGORIO } \\
\text { HERNÁNDEZ }\end{array}$ & 3 & 3 & 15 & 18 \\
\hline 1 & $\begin{array}{c}\text { Sucre, } \\
23 \text { de Enero }\end{array}$ & $\begin{array}{l}\text { UED.ALEJANDRO } \\
\text { FUENMAYOR }\end{array}$ & 2 & 2 & 22 & 10 \\
\hline Total & & & 17 & 17 & 308 & 120 \\
\hline
\end{tabular}

Fuente: Nómina de la Coordinación de Comunidad Escuela de la Secretaría para la Educación del Gobierno del Distrito Capital (2019)

La técnica fue la encuesta y el instrumento el cuestionario, el cual se elaboró en función de la Tabla de operacionalización de variables, y en su primera versión contaba con 60 ítems; la primera parte con respuesta en escala de frecuencia (siempre, algunas veces y nunca) y 8 ítems la segunda parte con respuesta de opinión de estos sobre el tema específico.

El instrumento fue validado por juicio de expertos constituidos por un metodólogo y tres profesionales de la gerencia. Con las observaciones se reformuló y se administró en una prueba piloto. Al resultado de esta prueba se le extrajo el índice de confiabilidad Alpha de Crombach, el cual fue de o,8o.

\section{RESULTADOS}

Se presenta el análisis porcentual o estadístico a partir de las repuestas dadas por los docentes con función de director y de los docentes de aula, tomando como referencia las tres dimensiones: personal, profesional e institucional, con sus respectivos indicadores (liderazgo, motivación, comunicación, manejo de conflicto y relaciones interpersonales para la dimensión personal; planificación, organización, control, ejecución y toma de decisiones para la dimensión profesional; sistema de recompensa y evaluación de desempeño para la dimensión institucional), señalados ya en la Tabla 1. Esto permitió el análisis y la discusión de los resultados entre lo encontrado y los autores consultados.

Para el análisis porcentual se tomó como alternativa positiva la opción "Siempre" y como negativas "Algunas veces" y "Nunca", además se añadió "No respondió”.

\section{Indicador "Liderazgo"}

En el ítem 12 referido a si el supervisor fomenta la responsabilidad de nuevas tareas y labores del docente y del personal directivo para una acción pedagógica de calidad; la opinión de los entrevistados es que "Nunca" o "Algunas veces" se hace. (ver Tabla 3 ).

En el ítem 29 los sujetos respondieron que nunca o algunas veces lo permite, situación distinta a lo que plantea Bittel 
(1999), cuando dicen que el supervisor tiene que encontrar la manera de persuadir o insistir para que el grupo o equipo de trabajo actué de conformidad con los objetivos de la organización, además el supervisor debe permitirle a los subordinados establecer sus metas juntos y demostrarles que esos objetivos se deben alcanzar mediante la acción colectiva es decir trabajo en equipo.

Así mismo las respuestas no se asemejan a lo que expresa Fuguet (2006), quien asegura que la supervisión participativa permite el trabajo en equipo, con formas de pensar, decidir y actuar acordes con la mentalidad flexible; promoviendo el compromiso como fuerza motora de la revitalización escolar. Igualmente las respuestas contradicen lo que expresa Requeijo (2008), quien afirma que uno de los objetivos del supervisor es promover actividades destinadas a fortalecer el trabajo en equipo, compartir responsabilidades y de apoyo mutuo entre los maestros (ver Tabla 3).

En el ítem 32, que indaga si el supervisor propicia el desarrollo integral del docente a través de la supervisión, las respuestas se ubican en la alternativa "Nunca" y "Algunas veces", contrario a lo que plantea Requeijo (2008), quien recomienda que el supervisor a través de la supervisión contribuya al desarrollo integral (crecimiento personal y profesional) de los docentes para que aplique con eficiencia conceptos, técnicas y principios que deben utilizarse en los procesos educativos a fin de eliminar los resultados indeseables (ver Tabla 3).

En el ítem 48 que pregunta si el supervisor orienta la elaboración del reglamento interno del plantel, los entrevistados respondieron que "Nunca" o "Algunas veces" los orientan, esto no se relaciona con lo que refiere López (2013), en cuanto a que una de las funciones del supervisor de sector es orientar y la elaboración del mismo donde no exista (ver Tabla 3).

En el ítem 49 que se refiere si el supervisor orienta la actividades extraescolares, los sujetos respondieron que "Nunca" y "Algunas veces" lo hacen, esto no se corresponde con lo que expresado por Villegas (2002) al asegurar que una de las funciones técnico docente del supervisor es asesorar, orientar y motivar la formulación de planes, sugerir al docente las diferentes actividades que puedan proponerse para el logro de los objetivos y proponer el desarrollo de las actividades extraescolares las cuales deben responder a las necesidades económicas, culturales, la salubridad, la recreación así como los intereses de los niños a las clases del aula y laboratorio (ver Tabla 3).

Tabla 3. Indicador "Liderazgo"

\begin{tabular}{cccccccccc}
\hline Ítems & Siempre & $\mathbf{\%}$ & $\begin{array}{c}\text { Algunas } \\
\text { veces }\end{array}$ & $\mathbf{\%}$ & Nunca & $\mathbf{\%}$ & $\begin{array}{c}\text { No } \\
\text { respondió }\end{array}$ & $\mathbf{\%}$ & Total \% \\
\hline 12 & 13 & 9,48 & 44 & 32,11 & 71 & 51,82 & 9 & 6,59 & 137.100 \\
29 & 19 & 13,86 & 38 & 27,73 & 66 & 48,17 & 14 & 10,24 & 137.100 \\
32 & 13 & 9,48 & 39 & 28,46 & 75 & 54,74 & 10 & 7,32 & 137.100 \\
48 & 15 & 10,94 & 39 & 28,46 & 72 & 52,55 & 11 & 8,05 & 137.100 \\
49 & 9 & 6,59 & 42 & 30,65 & 72 & 52,55 & 14 & 10,24 & 137.100 \\
\hline
\end{tabular}




\section{Indicador “Motivación”}

En el ítem 11, que pregunta si el supervisor orienta al logro de la metas, los informantes responden que "Nunca" o "Algunas veces" lo hacen. Esta respuesta no coincide con lo señalado por Ruiz (2005), quien opina que una de la funciones técnicodocente del supervisor es sugerir al docente las diferentes actividades y herramientas que pueda proponerse para el logro de los objetivos (ver Tabla 4 ).

En lo referente al ítem 22 que pregunta si el supervisor fomenta el desarrollo potencial del docente y del directivo, de igual manera las respuestas de los entrevistados es que "Nunca" o "Algunas veces" lo fomentan; lo que no se asemeja con la opinión de los autores consultados, en el caso de Fuguet (2006), cuando señala que el supervisor es un agente de transformación social, y debe preocuparse por fomentar la capacitación y desarrollo porcentual del docente. Para López (2013) una de las funciones del supervisor conjuntamente con el personal directivo es fomentar actividades que tiendan al mejoramiento profesional de los docentes (ver Tabla 4).

En el ítem 33, sobre si el supervisor maneja herramientas pedagógicas $y$ administrativas adecuadas para la orientación en el asesoramiento técnico, de igual manera los entrevistados respondieron que "Nunca" o "Algunas veces" lo hacen, esto no se asemeja con lo que expresan autores e investigadores analizados. Entre ellos Mogollón (2008) y Ruiz (2005), el primero de ellos recomienda en su investigación que el supervisor debe hacer uso de sus conocimientos y herramientas para mejorar la calidad de la gestión educativa y optimizar la supervisión escolar. Y el segundo plantea que el personal supervisor cada día debe prepararse más y conocer todas las herramientas necesarias para orientar $y$ estimular a sus subordinados en sus labores (ver Tabla 4).

Tabla 4. Indicador "Motivación"

\begin{tabular}{ccccccccccc}
\hline Ítems & Siempre & \% & $\begin{array}{c}\text { Algunas } \\
\text { veces }\end{array}$ & $\mathbf{\%}$ & Nunca & $\%$ & $\begin{array}{c}\text { No } \\
\text { respondió }\end{array}$ & $\%$ & Total \% \\
\hline 11 & 3 & 9,48 & 41 & 29,92 & 71 & 51,82 & 12 & 8,78 & 137100 \\
22 & 17 & 12,40 & 40 & 29,19 & 66 & 48,17 & 14 & 10,24 & 137100 \\
33 & 18 & 13,13 & 41 & 29,92 & 64 & 46,71 & 14 & 10,24 & 137100 \\
\hline
\end{tabular}

\section{Indicador "Comunicación”}

En el ítem 34, el cual expresa la interrogante si el supervisor propicia la interacción directivo-docente para mejorar el proceso de enseñanza aprendizaje, los entrevistados respondieron que "Nunca" o "Algunas veces", lo que difiere con lo que señalan autores tales como Bittel (1992; 1999) y Newstrom (1992) Estos consideran que el flujo de información invita a los compañeros de trabajo a la participación, el compromiso y al trabajo en equipo (ver Tabla 5).

En el ítem 36, que pregunta si el supervisor aplica la retroalimentación para mejorar los resultados de la gestión, los encuestados igual manera señalaron que "Nunca" o "Algunas veces" lo aplican. Según Hodgetts (1990), el supervisor tiene que aplicar la retroalimentación, por cuanto sirve 
para reforzar comportamiento y asegurar que el individuo vuelva a intentarlo nuevamente. También afirma que la comunicación oral es rápida y brinda la retroalimentación inmediata, es decir la comunicación frente a frente es el medio más eficiente (ver Tabla 5)

En el ítem 39 los entrevistados afirmaron que "Nunca" o "Algunas veces" lo hacen. Bittel (1999) señala que el supervisor, debe tener un buen trato con los demás, ya que las buenas relaciones son remunerativas (ver Tabla 5).

En el ítem 40 que cuestiona si el supervisor acepta con amabilidad las sugerencias del personal, y muestra interés en ella para establecer los cambios educativos en el plantel, los entrevistados respondieron "Nunca" o "Algunas veces". Esto contradice lo que expresa Hodgetts (1990), quien asegura que el supervisor debe ser un buen oyente, ya que la escucha activa implica eficacia, también incluye actitudes activas, empáticas y de apoyo que expresen al orador (ver Tabla 5).

En el ítem 50 que plantea si el supervisor se expresa con claridad y precisión, las respuestas de los entrevistados se inclinaron por las alternativas "Nunca" o "Algunas veces", esto no corresponde con lo que señala Bittel (1999), cuando señala que si quiere que los docentes tomen en serio lo que se le informan deben expresarse con seriedad, sin dejar dudas y seleccionar las palabras que transmitan claramente sus ideas y cuide su tono de voz (ver Tabla 5).

Tabla 5. Indicador "Comunicación”

\begin{tabular}{cccccccccc}
\hline Ítems & Siempre & $\mathbf{\%}$ & $\begin{array}{c}\text { Algunas } \\
\text { veces }\end{array}$ & $\mathbf{\%}$ & Nunca & $\%$ & $\begin{array}{c}\text { No } \\
\text { respondió }\end{array}$ & $\begin{array}{c}\text { Total } \\
\%\end{array}$ \\
\hline 34 & 13 & 9,48 & 40 & 29,19 & 74 & 54,01 & 10 & 7,32 & 137100 \\
36 & 14 & 10,24 & 43 & 31,38 & 69 & 50,36 & 11 & 8,05 & 137100 \\
39 & 30 & 21,89 & 41 & 29,92 & 56 & 40,87 & 12 & 8,78 & 137100 \\
40 & 20 & 14,62 & 47 & 34,30 & 59 & 43,06 & 11 & 8,05 & 137100 \\
50 & 26 & 18,97 & 47 & 34,30 & 51 & 37,22 & 13 & 9,48 & 137100 \\
\hline
\end{tabular}

\section{Indicador "Manejo de conflicto"}

En el ítem 18, que interroga si el supervisor induce a las personas generadoras de conflictos que expresan su malestar, las respuestas de los entrevistados se ubican en las alternativas "Nunca" o "Algunas veces", esto no se asemeja a lo que refieren Bittel y Newstrom (1992), sobre la importancia de solucionar conflictos al respecto refiere que en el conflicto está involucrados varios individuos, el supervisor tiene que invitarlos a una reunión y pida la participación para que manifiesten sus diferencia para así lograr un trabajo en armonía; "si puede buscar una persona que sirva de árbitro con autoridad que punta representar el punto de vista de la institución, pídale que se ponga en acción" (p. 353)

Por otra parte, las respuestas de los entrevistados son diferentes a lo que expresan Hodgetts (1990) y Dana (2002), al manifestar el primero que las diferencias de los grupos pueden explicarse con toda eficacia cuando se le pone en igualdad y minimizar las diferencias, si el supervisor tiene problemas con un subordinado o con el equipo de trabajo de la institución, debe buscar una escenario natural donde se pueda 
hablar con igualdad de condiciones. Y afirma que si el problema es más grave de lo esperado, el supervisor debe reunirse dos o tres veces con el grupo de trabajo y hasta acudir a instancias superiores para solicitar opinión y tal vez para que intervengan directamente tomando la decisión del caso. $Y$ el segundo afirma que el supervisor al utilizar la medición jerárquica, la misma es simple, esto en la práctica es difícil, porque hay que controlar impulsos e imponerse a algunos dictados del sentido común sobre la forma de ayudar a la gente a llegar a un acuerdo. Y añade que la mediación de equipo es una herramienta para resolver conflictos que tienen estructuras más complejas que las que se pueden resolver con medición jerárquica y automediación.

En lo referente al ítem 42, si el supervisor informa las irregularidades a las autoridades competentes, las repuestas de los entrevistados fueron "Nunca" o "Algunas veces", esto igualmente no corresponde con lo que señala López (2013) quien opina que una de la funciones del supervisor es elevar a la autoridades jerárquica competente o nivel superior del supervisor toda irregularidad y a la vez las medidas que consideren convenientes para subsanar las misma y cuando la solución no le corresponda en razón de su competencia (ver Tabla 6).

Tabla 6. Indicador "Manejo de conflicto"

\begin{tabular}{cccccccccc}
\hline Ítems & Siempre & $\%$ & $\begin{array}{c}\text { Algunas } \\
\text { veces }\end{array}$ & \% & Nunca & \% & $\begin{array}{c}\text { No } \\
\text { respondió }\end{array}$ & \% & Total \% \\
\hline 18 & 14 & 10,24 & 38 & 27,73 & 73 & 53,28 & 12 & 8,78 & 137100 \\
42 & 28 & 20,43 & 43 & 31,38 & 54 & 39,41 & 12 & 8,78 & 137100 \\
\hline
\end{tabular}

\section{Indicador "Relaciones interpersonales"}

En cuanto al ítem 17 sobre si el supervisor como líder educativo orienta a trabajar en armonía, las repuestas de los sujetos se orientaron a "Nunca" y "Algunas veces", por lo que difiere con lo que opina Smith (2003), al indicar que el supervisor descansa la responsabilidad de la orientación y lograr los beneficios cuando establece un ambiente amistoso, cuando proporciona información y cuando le expone los deberes y responsabilidades de los empleados (ver Tabla 7).

En lo referente al ítem 21, si el supervisor permite y fomente las relaciones entre los miembros de su equipo de trabajo, la respuestas de los sujetos se ubicaron en las alternativas "Nunca” y "Algunas veces", esto contradice lo que señala Bitell (1999), cuando habla de la necesidades sociales, al demostrar al respeto del grupo de trabajo y las necesidades de estimación, por lo que el supervisor debe hacer sentir a sus subordinados que su trabajo es valioso. Y que los supervisores deben respetar el poder y los intereses legítimos de los grupos de trabajos, sin abandonar las responsabilidades del grupo o sacrificando su propia autoridad, lo que permitirá que se alcancen los objetivos de la organización sin ignorar los derechos y expectativas de los equipos de trabajos (ver Tabla 7).

En el ítem 28, sobre si el supervisor motiva la interacción del personal a fin favorecer el logro de los objetivos, y el ítem 45 que refiere si el supervisor promueve actividades que mejoren las relaciones docentes alumnos, docente - docente y docente directivo, los encuestados aseguraron que nunca o algunas veces lo hacen, lo que no coincide con lo que señala Bitell (1999), el supervisor permite 
establecer metas y demostrar que esos objetivos se deben alcanzar mediante la interacción en el equipo de trabajo. De igual manera, el autor señala que la participación del personal determina la forma en que se debe realizar un trabajo, como debe dividirse el trabajo entre el grupo y cuáles son las metas de trabajo (ver Tabla 7).

Tabla 7. Indicador "Relaciones interpersonales"

\begin{tabular}{cccccccccc}
\hline Ítems & Siempre & $\%$ & $\begin{array}{c}\text { Algunas } \\
\text { veces }\end{array}$ & $\begin{array}{c}\text { Nunca } \\
\text { \% }\end{array}$ & $\begin{array}{c}\text { No } \\
\text { respondió }\end{array}$ & Total \% \\
\hline 17 & 22 & 16,05 & 42 & 30,65 & 60 & 43,76 & 13 & 19,48 & 137100 \\
21 & 17 & 12,40 & 43 & 31,38 & 64 & 46,71 & 13 & 19,48 & 137100 \\
28 & 18 & 13,13 & 37 & 27,0 & 69 & 50,36 & 13 & 19,48 & 137100 \\
45 & 16 & 11,67 & 37 & 27,0 & 72 & 52,55 & 12 & 18,78 & 137100 \\
\hline
\end{tabular}

\section{Indicador "Planificación"}

En cuanto al ítem 3, que cuestiona si el supervisor estimula al personal docente para la elaboración de la planificación, los entrevistados se ubicaron en las alternativas "Nunca" y "Algunas veces", lo que difiere de Requeijo (2008), quien plantea que sin planificación no se logran los objetivos, y a través de ella el docente organiza los objetivos, estrategias, recursos y actividades de evaluación de acuerdo al diagnóstico del aula, plantel y comunidad (ver Tabla 8).

Con respecto al ítem 4, sobre si el supervisor asesora la formulación de los planes del plantel, los encuestados respondieron que "Nunca" o "Algunas veces" lo hacen, esto difiere de los que señalan los autores Bittell y Neswstrom(1992) y Ruiz (2005), los primeros afirman que el supervisor debe estimular un plan integral dirigido en forma directa a su metas, pero si este se desvía, el supervisor debe tomar medidas correctivas para volverlo a su ruta, los cuales le proporcionarán la retroalimentación necesaria para poner en marchas las acciones. Y la segunda plantea que una de las funciones técnico- docente del supervisor es asesorar la formulación de planes del plantel, tanto del personal directivo como del docente de aula, basados éstos en el análisis de la situación real del diagnóstico (ver Tabla 8).

En lo que refiere al ítem 5, si el supervisor sugiere actividades complementarias que ayuden a los procesos de enseñanza y aprendizaje, los encuestados respondieron que "Nunca" o "Algunas veces", esto difiere de Ruiz (200o) cuando señala que una de las funciones técnico docente es sugerir al docente el desarrollo de actividades complementarias a las clases impartidas en el aula o laboratorio como: talleres, seminarios, foros, visitas a sitios de interés, organización de exposiciones, carteleras y preparación e actos culturales $\mathrm{u}$ otros relacionados con el medio educativo (ver Tabla 8).

En el ítem 8, que pregunta si el supervisor elabora planes de contingencias para atender eventualidades, los entrevistados se ubicaron en la alternativa nunca y algunas veces los hacen, esto coincide con lo que expresa Hodgetts (1990),ya que no siempre las cosas funcionan de acuerdo a los planes y la mayoría de las veces los supervisores no formulan planes de 
contingencias formales, simplemente piensa lo que tienen que hacer, si ciertas cosas no funcionan según lo previsto, los cuales se basan en consideraciones técnicas $y$ humanas (ver Tabla 8).

En el ítem 15, que refiere si el supervisor orienta al docente en los procedimientos pedagógicos según el currículo vigente, los entrevistados respondieron que nunca $y$ algunas veces lo hacen, esto difiere con lo que expresan Fuguet (2006), y Rutter y Conde (1998), el primero señala que la orientación al personal en los procedimientos del currículo vigente es necesaria y pertinente para satisfacer las necesidades de los alumnos en una sociedad especifica. Y las segundas refieren que la orientación curricular debe apuntar hacia la integración; adaptar estrategias y contenidos de los proyectos curriculares al contexto histórico y cultural en que se vive (ver Tabla 8).

En la pregunta 47, que señala si el supervisor asesora el funcionamiento de la comunidad educativa, los encuestados respondieron que "Nunca" o "Algunas veces". Para López (2013) y Ruiz (2000) las funciones técnico administrativa del supervisor deben ser asesorar y orientar la constitución y funcionamiento de las comunidades educativas de los planteles oficiales y privados (ver Tabla 8).

Tabla 8. Indicador "Planificación"

\begin{tabular}{cccccccccc}
\hline Ítems & Siempre & \% & $\begin{array}{c}\text { Algunas } \\
\text { veces }\end{array}$ & \% & Nunca & \% & $\begin{array}{c}\text { No } \\
\text { respondió }\end{array}$ & \% & Total \% \\
\hline 3 & 8 & 6,86 & 32 & 23,35 & 86 & 62,77 & 11 & 8,05 & 137100 \\
4 & 13 & 9,48 & 46 & 33,57 & 69 & 50,36 & 9 & 6,56 & 137100 \\
5 & 12 & 8,78 & 36 & 26,27 & 78 & 56,93 & 11 & 8,05 & 137100 \\
7 & 5 & 3,64 & 41 & 29,92 & 82 & 59,85 & 9 & 6,56 & 137100 \\
8 & 6 & 4,37 & 42 & 30,65 & 78 & 56,93 & 11 & 8,05 & 137100 \\
15 & 11 & 8,05 & 36 & 26,27 & 77 & 56,20 & 13 & 9,48 & 137100 \\
47 & 19 & 13,86 & 34 & 24,81 & 74 & 54,01 & 10 & 7,32 & 137100 \\
\hline
\end{tabular}

\section{Indicador "Organización”}

En cuanto al ítem 38 que refiere si el supervisor está atento a las funciones administrativas del docente, los encuestados respondieron que "Nunca" o "Algunas veces", lo que difiere con lo que señala Ruiz (200o) porque el supervisor debe prestar asesoría técnico administrativo al docente que coadyuven a lograr los objetivos y metas los cuales vayan en beneficio de la formación del alumno (ver Tabla 9).

En el ítem 43 referente a si el supervisor promueve el desarrollo de talleres y charlas informativas dirigidas a padres $y$ representantes, los encuestados respondieron que "Nunca" o "Algunas veces", esto difiere con lo que señala Fuguet (2006), ya que para él el supervisor es un constructor, creador, orientador de los demás miembros de la comunidad, apoya al personal docente en la producción de materiales, en la integración de equipo, la preparación de talleres de reflexión e intercambios y de capacitación para el personal, a los padres y representantes y demás miembros de la escuela (ver Tabla 9). 
Tabla 9. Indicador "Organización"

\begin{tabular}{ccccccccccc}
\hline Ítems & Siempre & $\%$ & $\begin{array}{c}\text { Algunas } \\
\text { veces }\end{array}$ & $\%$ & Nunca & $\%$ & $\begin{array}{c}\text { No } \\
\text { respondió }\end{array}$ & $\%$ & Total & $\%$ \\
\hline 38 & 20 & 14,59 & 37 & 27,0 & 70 & 51,09 & 10 & 7,32 & 137 & 100 \\
43 & 27 & 19,70 & 42 & 30,65 & 56 & 40,87 & 12 & 8,78 & 137 & 100 \\
\hline
\end{tabular}

\section{Indicador "Control"}

En lo que refiere al ítem 6 que indaga si el supervisor utiliza criterios técnicos para la elaboración de los recaudos administrativos, los encuestados respondieron que "Nunca" o "Algunas veces". Esto difiere de lo que señala Bittel (1999), quien afirma que las normas se establecen para guiar y tienen las responsabilidad de actuar dentro de los límites de las criterios técnicos administrativos o políticas educativas (ver Tabla 10).

En el ítem 9 sobre si el supervisor evalúa diferentes aspectos (planificación, evaluación, aplicación de estrategias) del desempeño laboral del personal docente y directivo, los encuestados respondieron que "nunca" o "Algunas veces", esto difiere de lo que afirma Requeijo (2008), quien dice que los supervisores revisan el trabajo que otros hacen, por cuanto participa en el control y evaluación de los procesos educativos en todos sus niveles (ver Tabla 10).

En cuanto al ítem 13 sobre si el supervisor utiliza criterios técnicos para mejorar el desempeño del personal docente y directivo, los encuestados respondieron que "Nunca" o "Algunas veces", esto no coincide con lo que expresa Requeijo (2008), ya que el supervisor debe utilizar criterios técnicos para suministrar orientaciones precisas de orden pedagógico, metodológico, técnico, administrativo y legal al personal docente en servicio para sí garantizar el éxito en la gestión educativa (ver Tabla 10).

En el ítem 19 sobre si la acción supervisora permite detectar la insuficiencias de la planta física, los entrevistados respondieron que "Nunca" o "Algunas veces", esto difiere de los que señala Ruiz (2000) y López (2013), cuando señala que una de las funciones técnico administrativa del supervisor es detectar las insuficiencias de la planta física, a fin de dar orientación al mantenimiento y elevar a las autoridades competentes informe de las deficiencias de infraestructura (ver Tabla 10).

En el ítem 20, que indaga si la supervisora permite que los recursos sean repartidos equitativamente, los encuestados respondieron que nunca o algunas veces, esto difiere de lo que expresa Fuguet (2006), ya que el supervisor debe gestionar los recursos financieros, humanos y materiales para ponerlo en disposición de la escuela y a su vez de los proyectos escolares, los cuales deben ser repartidos equitativamente según las necesidades (ver Tabla 10).

En el ítem 26, si el supervisor realiza seguimientos a los proyectos escolares, los entrevistados respondieron que nunca $o$ algunas veces, esto difiere de lo señalado por Fuguet (2006). El autor sostiene que el supervisor debe hacerle seguimiento a los proyectos escolares ya que la supervisión ayuda a planificar mejor tales proyectos y potenciarlos como una estrategia que permita fortalecer la gestión escolar, además se fundamentan en concepto como aprendizaje significativo del alumno y se orienta hacia la actualización del personal y la selección de estrategias que definen el estilo de gestión propia de cada escuela (ver Tabla 10). 
Tabla 10. Indicador "Control"

\begin{tabular}{lcccccccccc}
\hline Ítems & Siempre & $\%$ & $\begin{array}{c}\text { Algunas } \\
\text { veces }\end{array}$ & $\mathbf{\%}$ & Nunca & $\mathbf{\%}$ & $\begin{array}{c}\text { No } \\
\text { respondió }\end{array}$ & \% & Total & $\%$ \\
\hline 6 & 20 & 14,59 & 41 & 29,92 & 64 & 46,71 & 12 & 8,78 & 137 & 100 \\
9 & 19 & 13,86 & 39 & 28,46 & 70 & 51,09 & 9 & 6,56 & 137 & 100 \\
13 & 16 & 11,67 & 48 & 35,03 & 62 & 45,25 & 11 & 8,05 & 137 & 100 \\
19 & 20 & 14,59 & 35 & 25,54 & 67 & 48,90 & 15 & 10,94 & 137 & 100 \\
20 & 17 & 12,40 & 38 & 27,73 & 70 & 51,09 & 12 & 8,78 & 137 & 100 \\
26 & 16 & 11,67 & 43 & 31,38 & 67 & 48,90 & 11 & 8,05 & 137 & 100 \\
\hline
\end{tabular}

\section{Indicador "Ejecución”}

En relación con el ítem 10, que se pregunta si el supervisor realiza acompañamiento pedagógico, los entrevistados respondieron que "Nunca" o "Algunas veces", esto difiere de lo que señalan Fuguet (2006) y Requeijo (2008) el primero afirma que la supervisión participativa debe acompañar y apoyar las prácticas pedagógicas en el aula. $\mathrm{Y}$ el segundo señala que la visita de aula de clases o acompañamiento pedagógico por parte del supervisor es necesaria y de carácter institucional que reviste interés especial ya que de ella depende en alto grado de calidad de la enseñanza ya que se corrige las desviaciones que puedan detectarse (ver Tabla 11).

En el ítem 24 sobre si el supervisor evalúa los aspectos del desempeño de su personal docente y directivo. Los encuestados respondieron que "Nunca" o "Algunas veces", en este sentido Requeijo (2008) afirma que es necesaria evaluar el desempeño del personal de las instituciones (directivos y docente), con la finalidad de corregir las discrepancias de lo previsto en el plan o el proyecto y lo alcanzado en la realidad (ver Tabla 11).
En el ítem 35 sobre si el supervisor aplica las leyes y aspectos jurídicos relacionados con la supervisión y el sistema educativo, los entrevistados respondieron que "Nunca" o "Algunas veces", esto difiere de lo que afirman López (2013), Mogollón (2008) y Ruiz (2000) quienes coinciden que una de las funciones del supervisor es conocer y aplicar todos los aspectos y normativas jurídicas a fin de aplicar en el momento que sea necesario en su gestión educativa (ver Tabla 11).

Y sobre si el supervisor evalúa conjuntamente con el personal docente el impacto del proyecto educativo integral comunitario (ítem 44), los encuestados respondieron que nunca o algunas veces, en este sentido Fuguet (2006) a firma que lograr el aprendizaje producto de las experiencias educativas se relacionan con los propósitos comunes y la visión compartida de la comunidad comprometida con el proyecto educativo integral comunitario a fin de logra mantener la excelencia pedagógica del plantel y el aprendizaje de los alumnos (ver Tabla 11). 
Tabla 11. Indicador "Ejecución"

\begin{tabular}{ccccccccccc}
\hline Ítems & Siempre & $\mathbf{\%}$ & $\begin{array}{c}\text { Algunas } \\
\text { veces }\end{array}$ & $\mathbf{\%}$ & Nunca & $\mathbf{\%}$ & $\begin{array}{c}\text { No } \\
\text { respondió }\end{array}$ & $\mathbf{\%}$ & Total & $\%$ \\
\hline 10 & 10 & 7,32 & 37 & 27,00 & 79 & 57,66 & 11 & 8,05 & 137 & 100 \\
24 & 24 & 17,51 & 40 & 29,19 & 61 & 44,52 & 12 & 8,78 & 137 & 100 \\
35 & 21 & 15,32 & 40 & 29,19 & 65 & 47,44 & 11 & 8,05 & 137 & 100 \\
44 & 17 & 12,40 & 38 & 27,73 & 71 & 51,82 & 11 & 8,05 & 137 & 100 \\
\hline
\end{tabular}

\section{Indicador "Toma de decisiones"}

En cuanto al ítem 16 que pregunta cuando surgen problemas con el personal de la institución el supervisor solicita ideas y opiniones de los docente para solucionarlos, los encuestados respondieron que "Nunca" o "Algunas veces", esto difiere de lo que afirma Ruiz (2000), cuando recomienda que se le pida a los empleados que presente una lista de alternativas para solucionar la problemática presentada y es factible que entre ellos encuentren soluciones pertinentes y de esa manera se propicia la participación y la integración de todos el procesos educativo (ver Tabla 12).
En el ítem 23 sobre si el supervisor permite la libertad en la toma de decisiones que atañan directamente en sus labores, los sujetos encuestados respondieron que nunca o algunas veces, esto difiere de lo que afirma Ruiz (2000), en el campo de la educación se facilita la toma de decisiones, por cuanto la mayoría de las situaciones están pautadas en leyes, reglamentos, la toma de decisiones es realizada por el personal directivo, subdirectores, coordinadores y docentes de aula, en este sentido también se le pide elabore posible alternativas a fin de considerarla y tomar la decisión respectiva (ver Tabla 12).

Tabla 12. Indicador "Toma de decisiones"

\begin{tabular}{lcccccccccc}
\hline Ítems & Siempre & $\mathbf{\%}$ & $\begin{array}{c}\text { Algunas } \\
\text { veces }\end{array}$ & $\mathbf{\%}$ & Nunca & $\mathbf{\%}$ & $\begin{array}{c}\text { No } \\
\text { respondió }\end{array}$ & $\mathbf{\%}$ & Total & $\%$ \\
\hline 16 & 14 & 10,24 & 40 & 29,19 & 70 & 51,09 & 13 & 9,48 & 137 & 100 \\
23 & 20 & 14,59 & 45 & 32,84 & 58 & 42,33 & 14 & 10,24 & 137 & 100 \\
\hline
\end{tabular}

\section{Dimensión institucional}

\section{Indicador "Sistema de recompensa"}

En cuanto al ítem referido al sistema de recompensa, se observa que el 68,63\% señaló que no se dispone de este. Según Requeijo (2008), en todas organizaciones educativas debe existir la promoción de empleados que implica lo concerniente a la mejora en la remuneración y la posibilidad de apoyo para el mejoramiento del desempeño a fin de adquirir nueva habilidades (ver Tabla 13).

Tabla 13. Indicador "Sistema de recompensa

\begin{tabular}{lllllllll}
\hline Ítem & SI & \% & NO & \% & NR & \% & Total & $\%$ \\
\hline 1 & 19 & 13,86 & 94 & 68,63 & 24 & 17,51 & 137 & 100 \\
\hline
\end{tabular}




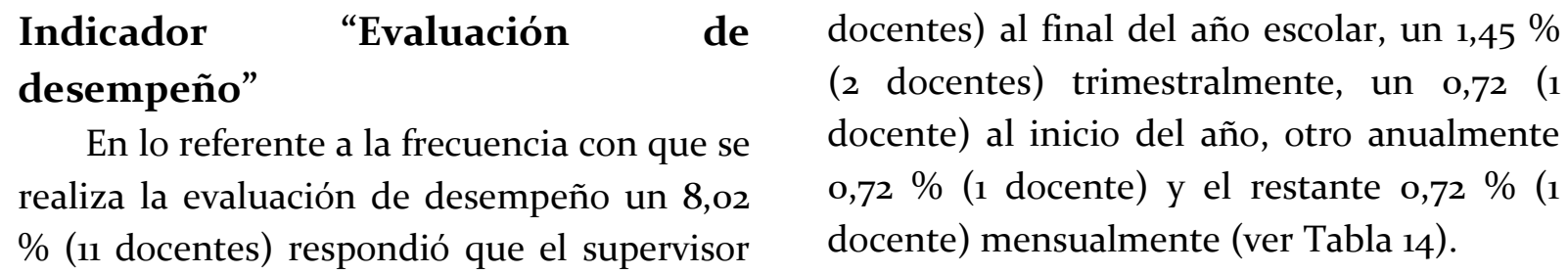

la realiza durante el año escolar, un 2,18 \% (3

Tabla 14. Frecuencia con la que se realiza la evaluación de desempeño

\begin{tabular}{llllllllllllllllll}
\hline Ítems & $\mathbf{1}$ & $\mathbf{\%}$ & $\mathbf{2}$ & $\mathbf{\%}$ & $\mathbf{3}$ & $\mathbf{\%}$ & $\mathbf{4}$ & $\mathbf{\%}$ & $\mathbf{5}$ & $\mathbf{\%}$ & $\mathbf{6}$ & $\mathbf{\%}$ & $\mathbf{7}$ & $\mathbf{\%}$ & Total & $\mathbf{\%}$ \\
\hline 2 & 1 & 0,72 & 11 & 8,02 & 1 & 0,72 & - & - & 2 & 1,45 & 1 & 0,72 & 3 & 2,18 & 19 & 13,86 \\
\hline
\end{tabular}

Nota: 1) al inicio del año escolar; 2) durante el año escolar; 3) anual; 4) semestral; 5) trimestral; 6) mensual; 7) al final del año escolar.

En cuanto a si el docente conoce el resultado de la evaluación de desempeño, la mayoría de los encuestados "No respondió" $(43,79 \%)$, esto difiere con lo que señalan Bittel y Newstrom (1992), cuando afirman que el personal tiene derecho a saber el resultado de su evaluación y desean saber además que tan bien realizan su trabajo (ver Tabla 15).

Tabla 15. Frecuencia con la que se informa el resultado de la evaluación de desempeño

\begin{tabular}{ccccccccccc}
\hline Ítems & $\mathbf{1}$ & $\mathbf{\%}$ & $\mathbf{2}$ & $\mathbf{\%}$ & $\mathbf{3}$ & $\mathbf{\%}$ & $\mathbf{N R}$ & $\mathbf{\%}$ & Total & $\mathbf{\%}$ \\
\hline 5 & 49 & 35,79 & 9 & 6,56 & 19 & 13,86 & 60 & 43,79 & 137 & 100 \\
\hline
\end{tabular}

Nota: 1) Escrita; 2) Oral; 3) Todas las anteriores; NR) no respondió

CONCLUSIONES

Existe un alto porcentaje de debilidades en los procesos gerenciales que realizan los supervisores de las escuelas que dependen de la Secretaría para la Educación del Gobierno del Distrito Capital, debido a que no cumplen las funciones que les corresponden, no planifican, el liderazgo es débil, no motivan, no hay comunicación y no organizan.

Con respecto a la aplicación de la acción supervisora, se evidencia deficiencia, ya que los procesos gerenciales como orientación, asesoramiento, motivación y toma de decisiones se cumplen en forma incorrecta y en la mayoría de los casos no existe.
En cuanto a las funciones de asesoramiento pedagógico, relaciones interpersonales e institucionales y manejo de conflicto, también hay debilidades y existen discrepancias en el asesoramiento que reciben los directores y docentes de los planteles por parte del supervisor.

\section{REFERENCIAS}

Arias, F. (2006). El proyecto de Investigación. Guía para su elaboración. Caracas: Editorial Episteme CA/Orial Ediciones

Ayala, E. (2014). Manual de Supervisión Educativa. Santo Domingo, Distrito Nacional 
Bittel, L. y Newstrom, J. (1992). Lo que todo supervisor debe saber. (6ta Ed.) México: McGraw-Hill Interamericana de México

Bittel L. (1999). Curso Practico MrMcgrawhill del Supervisor. Colombia: Impreandes Presencia S.A

Borjas U., F. J. y Vera G., M. B. (2008). Funciones gerenciales del director de las escuelas bolivarianas. NEGOTIUM. Revista Científica Electrónica Ciencias Gerenciales, 11(4), 70-103 Recuperado de www.revistanegotium.org.ve

Bringuier, L. (2001). La Investigación Educativa como Proceso de Formación Permanente. Instituto Pedagógico Latinoamericano y Caribeño. La Habana Cuba. Recuperado de www.grupocieg.org

Chiavenato, I. (2006). Administración. Proceso administrativo. España: Editorial Mc Graw Hill

Dana, D. (2002). Adiós a los conflictos. España: Editora María Victoria Peralba Ruiz

Esteban F., S. (2019). La supervisión educativa como función principal de la inspección. Características y propósitos. Aula, 25, 27-58. Recuperado de https://revistas.usal.es/index.php/o2143402/article/view/aula2019252758/20794

Fuguet, A. (2006). Supervisión Participativa y Proyectos Escolares. Caracas: Vicerrectorado de Investigación y Postgrado, UPEL

García, B. (2015). La supervisión educativa ¿víctima o excluida de las políticas públicas. Recuperado de https://docplayer.es/66752804-Lasupervision-educativa-victima-oexcluida-de-las-politicas-publicas.html

Hodgetts, R. M. (1990). El supervisor eficiente, un enfoque práctico. Impreso en México

Hurtado, I. y Toro (1998). Paradigma y Métodos de Investigación en Tiempos de Cambios (2da edición). Valencia, Venezuela: Clemente Editores C.A

Lemus, L. (2005). Administración, dirección y supervisión de escuelas. Argentina: Editorial Kapelusz

López, R. (2013). Manual del Supervisor, Director y Docente. Ed. Monfort C.A

López, R. (2012). Nuevo Manual del Supervisor, Director y Docente. Caracas: Editorial Torino
Maldonado, C. (2008). Evaluación de Desempeño Docente en el Proceso de Enseñanza- Aprendizaje de los Estudios Básicos Supervisados del Instituto Universitario Tecnológico de Maracaibo (trabajo de maestría). Universidad Nacional Abierta (UNA), Venezuela Mendoza, A., I. (2018). Supervisión educativa en la Educación Básica. Revista Arbitrada del CIEG, Centro de Investigación y Estudios Gerenciales, 34 octubre-diciembre 2018, 172-189. Recuperado de www.grupocieg.org

Mogollón, A. (2008). Calidad y enfoque de la Supervisión. Revista de Educación, Universidad de Carabobo, Facultad de Ciencias de la Educación ValenciaVenezuela

Ocando C., H. Y. (2017). La supervisión educativa como elemento clave para alcanzar la calidad educativa en las escuelas públicas. Omnia, 23(3), 42-57

Requeijo, D. (2008). Administración y gerencia. Miranda, Venezuela: Ed. Biosfera

Ruiz, J. (1992). Gerencia en el aula. Caracas: FEDEUPEL

Ruiz, N. (2000). La gerencia y el proceso de la educación. Caracas: UPEL- Instituto Pedagógico de Caracas. Departamento de Pedagogía

Ruiz, N. (2005). Aproximación a un Modelo Teórico de Supervisión Escolar para Propiciar la Excelencia de la Educación (tesis doctoral). Universidad Santa María, Caracas, Venezuela

Rutter I. y Conde M (1998). La Supervisión Educativa Gerencia y Pedagogía. Caracas: Fondo Editorial FEDUPEL

Smith, C. (2003). Guías para Supervisores (5 Ed). México: Editorial Trillas

Universidad Pedagógica Experimental Libertador. Vicerrectorado de Investigación y Postgrado. (2017).Manual de Trabajo de Grado de Especialización y Maestría y Tesis Doctorales. Caracas, Venezuela: FEDUPEL

Villegas (2002). Gerencia y liderazgo (trabajo de grado). Instituto Pedagógico de Barquisimeto Luis Beltrán Prieto Figueroa, Venezuela: UPEL 
Mendoza, A., I. (2018). Supervisión educativa en la Educación Básica. Revista Arbitrada del CIEG, Centro de Investigación y Estudios Gerenciales, 34 octubrediciembre 2018, 172-189. Recuperado de www.grupocieg.org

Mogollón, A. (2008). Calidad y enfoque de la Supervisión. Revista de Educación, Universidad de Carabobo, Facultad de Ciencias de la Educación ValenciaVenezuela

Ocando C., H. Y. (2017). La supervisión educativa como elemento clave para alcanzar la calidad educativa en las escuelas públicas. Omnia, 23(3), 42-57

Requeijo, D. (2008). Administración y gerencia. Miranda, Venezuela: Ed. Biosfera

Ruiz, J. (1992). Gerencia en el aula. Caracas: FEDEUPEL

Ruiz, N. (2000). La gerencia y el proceso de la educación. Caracas: UPEL- Instituto
Pedagógico de Caracas. Departamento de Pedagogía

Ruiz, N. (2005). Aproximación a un Modelo Teórico de Supervisión Escolar para Propiciar la Excelencia de la Educación (tesis doctoral). Universidad Santa María, Caracas, Venezuela

Rutter I. y Conde M (1998). La Supervisión Educativa Gerencia y Pedagogía. Caracas: Fondo Editorial FEDUPEL

Smith, C. (2003). Guías para Supervisores (5 Ed). México: Editorial Trillas

Universidad Pedagógica Experimental Libertador. Vicerrectorado de Investigación y Postgrado. (2017).Manual de Trabajo de Grado de Especialización y Maestría y Tesis Doctorales. Caracas, Venezuela: FEDUPEL

Villegas (2002). Gerencia y liderazgo (trabajo de grado). Instituto Pedagógico de Barquisimeto Luis Beltrán Prieto Figueroa, Venezuela: UPEL 